16,01

\title{
Моделирование взаимодействия двудольных биметаллических кластеров с низкоэнергетическими кластерами аргона
}

\author{
(ㄱ Д.В. Широкорад ${ }^{1}$, Г.В. Корнич ${ }^{1}$, С.Г. Буга ${ }^{2,3}$ \\ ${ }^{1}$ Запорожский национальный технический университет, \\ Запорожье, Украина \\ ${ }^{2}$ Технологический институт сверхтвердых и новых углеродных материалов, \\ Москва, Троицк, Россия \\ ${ }_{3}^{3}$ Московский фризико-технический институт (Государственный университет), \\ Долгопрудный, Россия \\ E-mail: slejpnir@zntu.edu.ua
}

(Поступила в Редакцию 22 декабря 2015 г.

В окончательной редакции 26 мая 2016 г.)

\begin{abstract}
Выполнено молекулярно-динамическое моделирование эволюции двудольных биметаллических кластеров, состоящих из 390 атомов, при бомбардировке кластерами $\operatorname{Ar}_{n}(n=1,2,13)$ с начальной энергией от $1 \mathrm{eV}$ до $1.4 \mathrm{keV}$. В качестве мишени использовались бинарные кластеры $\mathrm{Cu}-\mathrm{Au}$ и $\mathrm{Ni}-\mathrm{Al}$, состоящие из равных по количеству атомов долей соответствующих элементов. В результате моделирования получены температуры, изменения потенциальной энергии, выходы распыления, а также интенсивности столкновительно- стимулированных перемещений атомов через границу раздела монометаллических частей бинарных кластеров в зависимости от энергии и размера налетающей частицы.
\end{abstract}

Работа выполнена в рамках соглашения № 14.580.21.0003 (уникальный идентификатор проекта RFMEFI58015X0003) при финансовой поддержке Министерства образования и науки РФ (С.Г.Б.).

DOI: 10.21883/FTT.2017.01.43973.480

\section{1. Введение}

В эпоху миниатюризации электронных устройств возрастает практическая необходимость в исследовании свойств наноразмерных атомных структур [1,2]. В частности, проблематика исследования и применения атомных нанокластеров охватывает области физики, химии, материаловедения, биологии, формируя отдельную на сегодняшний день отрасль знаний. При этом, объектами пристального внимания как с практической, так и с академической точек зрения являются металлические кластеры [3]. Среди посвященных им исследований существует много работ по изучению пар компонентов кластеров $\mathrm{Cu}-\mathrm{Au}[4-6]$ и $\mathrm{Ni}-\mathrm{Al}[7,8]$. Их форма, атомная структура, композиция и сегрегационные свойства являются объектами исследования, поскольку они влияют на химическую активность и каталитические свойства $[9,10]$. Было также замечено, что некоторые пары металлов в кластерах проявляют критически отличную от макроскопических образцов вещества склонность к взаимному перемешиванию [11]. Биметаллические нанокластеры имеют практическое значение для формирования токовых и потенциальных контактов в элементах электроники в связи с тем, что работа выхода электронов сильно зависит от реальной структуры нанокластеров. Кроме того, тонкие покрытия $\mathrm{Cu}-\mathrm{Au}$ и $\mathrm{Ni}-\mathrm{Al}$ активно используются для реактивного химического сращивания (бондинга) полупроводниковых структур в многослойные интегральные схемы с целью повышения плотности упаковки отдельных элементов за счет реализации трехмерной архитектуры [12-14].
Теоретические подходы к изучению эволюции структуры и физико-химических свойств кластеров основаны как на фундаментальных квантовохимических методах, так и на феноменологических представлениях [15]. Обзор экспериментальных методов исследования атомных кластеров, в том числе способов их получения, представлен в работах [16,17]. Наряду с теоретическими и экспериментальными подходами широко применяются также различные виды компьютерного моделирования, в частности метод классической молекулярной динамики [18-20].

В течение последних пятнадцати лет метод классической молекулярной динамики успешно использовался для моделирования процессов дефектообразования в свободных атомных кластерах, а также их распыления под действием энергетических ионов [21-27]. Рассматривались однокомпонентные кластеры разных размеров, включая большие кластеры с числом атомов до $10^{6}$, которые подвергались бомбардировке одноатомными ионами с энергиями до deca-keV-ных величин. Было показано, что выходы распыления, включающие высокоэнергетическую каскадно-рекойловую и низкоэнергетическую термическую составляющие, демонстрируют значения, близкие к максимальным, и существенно превышают выходы распыления для плоской поверхности в случае, когда характерный размер кластера (радиус при его сферическом представлении) согласуется со средней глубиной распределения потерь энергии бомбардирующих атомов.

Очевидно, что использование атомных кластеров различных типов и размеров наряду с единичными атомами 
в качестве бомбардирующих частиц предоставит больше возможностей для варьирования объемного распределения энергетических потерь и передаваемого импульса в бомбардируемом кластере. Широкое распространение получило применение ионных пучков кластеров газовых атомов (GCIB) [28] для модификации/очистки поверхности, послойного анализа и осаждения тонких пленок, берущее начало с классической работы [29]. Однако и сейчас развитие, в том числе моделирование, методики GCIB, включая пучки кластеров аргона для обработки различных поверхностей, является технологически [30-32] и академически [33] актуальным. В настоящей работе исследованы свободные двудольные биметаллические кластеры $\mathrm{Ni}-\mathrm{Al}$ и $\mathrm{Cu}-\mathrm{Au}$, состоящие из 390 атомов, под действием бомбардировки моноэнергетическими кластерами аргона различных размеров, обладающими энергиями от $1 \mathrm{eV}$ до $1.4 \mathrm{keV}$. Настоящее исследование является дальнейшим развитием работы [34], где проводилось молекулярно-динамическое моделирование эволюции свободных двудольных биметаллических кластеров при их бомбардировке моноэнергетическими одноатомными ионами Ar. Использование небольших кластеров аргона в качестве бомбардирующих частиц позволяет получать большую энергоотдачу по сравнению с единичными атомами аргона в процессе столкновения с массивным биметаллическим кластером при равных достаточно больших начальных энергиях. Это дает возможность индуцировать критически более высокие температуры и большую интенсивность массопереноса атомов компонентов в объеме и на поверхности биметаллического кластера, а также демонстрировать существенно бо́льшие выходы распыления, в которых, помимо вклада каскадно-рекойловых механизмов в течение первых нескольких пикосекунд, может быть также и значительная термическая составляющая за счет испарения атомов с его поверхности, проявляющая себя на протяжении всего времени моделирования.

\section{2. Модельные представления}

Как и в работе [34], для получения двухкомпонентных модельных кластеров были выбраны две пары элементов: медь-золото и никель-алюминий. Помимо практической применимости, такой выбор обусловлен динамическими особенностями атомов компонентов и различной теплотой перемешивания $\left(\Delta H_{\{A B\}}^{m i x}\right)$ пар материалов $A$ и $B$, что позволяет выявить определенные закономерности в свойствах подобных бинарных наноструктур, связанные с перемешиванием атомов между двумя однокомпонентными долями в результате внешнего столкновительного воздействия. Известно, что для пары компонентов $\mathrm{Ni}-\mathrm{Al} \Delta H_{\{A B\}}^{\text {mix }}=-22 \mathrm{~kJ} / \mathrm{mol}$, тогда как для пары $\mathrm{Cu}-\mathrm{Au} \Delta H_{\{A B\}}^{\text {mix }}=-9 \mathrm{~kJ} / \mathrm{mol}$ [35], что свидетельствует о большей способности первой пары компонентов к взаимному перемешиванию.

Для получения двудольных модельных кластеров два исходных однокомпонентных кристалла разных метал- лов по 195 атомов в виде неполных прямоугольных параллелепипедов релаксировали отдельно друг от друга в течение 150 ps с непрерывной диссипацией кинетической энергии, выделяющейся в результате трансформации формы кристалла и соответствующего уменьшения свободной поверхности. Конечные однокомпонентные кластеры сохраняли ГЦК структуру и принимали форму неидеальных усеченных октаэдров с шестиугольными $\{111\}$ и квадратными $\{100\}$ гранями. Полученные однокомпонентные кластеры размещались случайным образом на расстоянии $2 \AA$ между их ближайшими поверхностными атомами. Под действием сил межатомного взаимодействия они сливались и релаксировали опять в течение $150 \mathrm{ps}$ с диссипацией избыточной кинетической энергии [36], образуя единый устойчивый двудольный кластер, состоящий из двух однокомпонентных долей с небольшим взаимным проникновением атомов на границе раздела между долями. В результате были получены два двудольных биметаллических кластера, состоящие из 390 атомов для каждой пары элементов [37], которые были использованы в дальнейших модельных экспериментах в качестве начальных бомбардируемых кластеров.

Бомбардировка полученных модельных бинарных кластеров проводилась кластерами $\mathrm{Ar}_{n}$, где $n=1,2,13$, которые располагались в случайно выбранных точках пространства так, что расстояние между ближайшими атомами мишени и кластера $\mathrm{Ar}_{n}$ составляло 6-7 Векторы скорости всех налетающих атомов аргона в начальный момент времени были одинаковыми по модулю и ориентированы параллельно вектору, направленному от центра масс кластера $\mathrm{Ar}_{n}$ в центр масс исходного двудольного кластера. Каждое испытание эволюции системы „двудольный кластер-бомбардирующий кластер“ выполнялось в течение 5 ps, что позволило корректно учесть каскадно-рекойловую часть столкновительного процесса, особенно при больших энергиях бомбардировки, вплоть до его затухания, и развитие термической стадии. Также были выполнены модельные испытания температуры и распыления кластера, включая термическую стадию, в течение 100 ps. Для каждой выбранной энергии бомбардирующего кластера аргона проводилось по 2000 испытаний для времени моделирования 5 ps и 200 испытаний для времени моделирования 100 ps. Каждое испытание отличалось начальными координатами бомбардирующей частицы и соответственно направлением вектора скорости в начальный момент времени (рис. 1).

Моделирование осуществлялось методом классической молекулярной динамики [38]. Для описания взаимодействия атомов металлов использовался многочастичный аналитический потенциал Финниса и Синклаира (Finnis and Sinclair) [39] в версии Акланда (Ackland) [40] с присоединенным к нему в области высоких энергий отталкивающим потенциалом Борна-Майера (Born-Mayer). Было принято во внимание, что потенциал Акланда реалистично описывает 
структуру однокомпонентных нанокластеров при различных температурах [41], а также механические свойства однокомпонентных и бинарных наночастиц $[42,43]$. Вычисленные согласно потенциалу Акланда теплоты перемешивания пар $\mathrm{Ni}-\mathrm{Al} \Delta H_{\{A B\}}^{m i x}=-18.3 \mathrm{~kJ} / \mathrm{mol}$ и $\mathrm{Cu}-\mathrm{Au} \Delta H_{\{A B\}}^{m i x}=-5.3 \mathrm{~kJ} / \mathrm{mol}$ качественно соответствуют приведенным выше экспериментальным значениям, что позволило оценивать влияние взаимного перемешивания компонентов кластеров на измеряемые параметры.

Взаимодействие атомов металл-аргон описывалось универсальным отталкивающим потенциалом Зиглера-Бирзака-Литтмарка (Ziegler-Biersack-Littmark $\mathrm{ZBL}$ ) [44]. Взаимодействия $\mathrm{Ar}-\mathrm{Ar}$ в данной модели задавались потенциалом HFDTCS1 [45], который образован путем объединения с помощью экспоненциальной функции связи потенциалов HFD-B3 [46] и Борна-Майера. Парный потенциал HFD-B3 имеет вид

$$
V(r)=\varepsilon V^{*}(x),
$$

где

$$
\begin{gathered}
V^{*}(x)=A^{*} \exp \left(-\alpha^{*} x+\beta^{*} x^{2}\right)-F(x) \sum_{j=0}^{2} c_{2 j+6} / x^{2 j+6} \\
F(x)= \begin{cases}\exp \left(-(D / x-1)^{2}\right), & x<D \\
1, & x \geq D\end{cases}
\end{gathered}
$$

Присоединяя потенциал Борна-Майера, получаем потенциал HFDTCS1

$$
\begin{gathered}
V^{*}(x)=B^{*} \exp \left(-b^{*} x\right), \quad x<x_{1}, \\
V^{*}(x)=\exp \left(a_{1}+\left(x-x_{1}\right)\left(a_{2}+\left(x-x_{2}\right)\right.\right. \\
\left.\left.\quad \times\left(a_{3}+\left(x-x_{1}\right) a_{4}\right)\right)\right), \quad x_{1} \leq x \leq x_{2}, \\
V^{*}(x)=V_{\mathrm{HFD}-\mathrm{B} 3}^{*}(x), \quad x>x_{2},
\end{gathered}
$$

где $x=r / r_{m}, r_{m}=3.761 \AA . \varepsilon, c_{i}, A^{*}, \alpha^{*}, \beta^{*}, D, b, B-$ известные параметры [45], $r_{1}$ и $r_{2}$ составляли 2.65 и $2.70 \AA$ соответственно. Коэффициенты $a_{i}$ рассчитываются из условий непрерывности и гладкости функции потенциала. Радиус обрезания потенциала взаимодействия $\mathrm{Ar}-\mathrm{Ar}$ составлял $5 \AA$, что позволило включить в расчет соответствующих уравнений движения его притягивающую часть и естественным образом моделировать размер кластера $\mathrm{Ar}_{13}$, имеющего форму икосаэдра.

Численное решение уравнений движения атомов осуществлялось методом Верле [47] с шагом по времени не более $0.5 \mathrm{fs}$. Ошибка выполнения закона сохранения энергии при этом была не более 3\%. Используемая для стабилизации структуры начального кластера диссипация кинетической энергии осуществлялась путем введения в уравнения движения Ньютона всех его атомов дополнительного слагаемого, пропорционального скорости движения атома и ориентированного против мгновенного направления его движения.

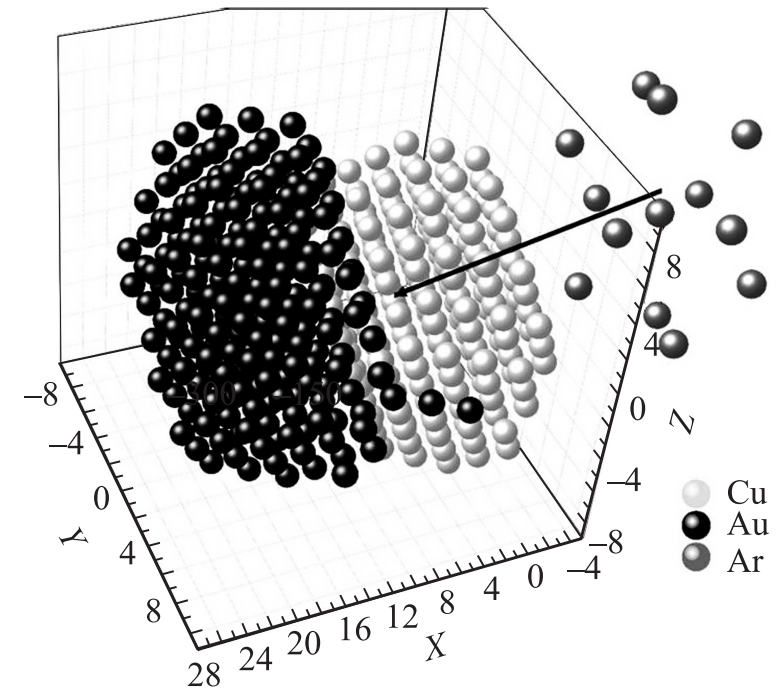

Рис. 1. Модельный кластер $\mathrm{Cu}-\mathrm{Au}$, состоящий из 390 атомов, и налетающий кластер $\operatorname{Ar}_{13}$ в начальный момент времени.

Температура кластера оценивалась согласно средней кинетической энергии его атомов относительно центра масс

$$
T=\sum_{i=1}^{N} \frac{m_{i}\left(v_{c . m .}-v_{i}\right)^{2}}{2} \cdot \frac{2}{3 N k_{B}},
$$

где $v_{c . m .}-$ скорость центра масс кластера, $N-$ число атомных частиц. Энергия распыленных атомов не учитывалась при вычислении температуры кластера. Как и в предыдущей модели [34], отвод энергии из бомбардируемого кластера, привносимой налетающим кластером аргона, был возможен только за счет самих атомов Ar, покидающих металлический кластер, а также распыленных в результате столкновительных взаимодействий атомов металлов.

\section{3. Результаты моделирования и обсуждения}

На рис. 2 приведены временны́е зависимости выхода распыления и температуры двудольных кластеров $\mathrm{Cu}-\mathrm{Au}$ и $\mathrm{Ni}-\mathrm{Al}$ при взаимодействии с энергетической частицей $\operatorname{Ar}_{n}(n=1,13)$ и полном времени эволюции 100 ps для иллюстрации каскадно-рекойловых столкновительных процессов и перехода к термической стадии каскада при различных энергиях бомбардировки. В случае бомбардировки двудольных кластеров единичными атомами Ar каскадно-рекойловое распыление завершается за первые 5 ps, после чего картина распыления критически меняется, и далее наблюдаются редкие события термического распыления слабосвязанных атомов двудольного кластера, которое можно трактовать как испарение для больших времен моделирования. Температура кластера имеет резкий пик в течение первой пикосекунды, что связано с генерацией высокоэнергетических атомов отдачи в кластерах с последующим 

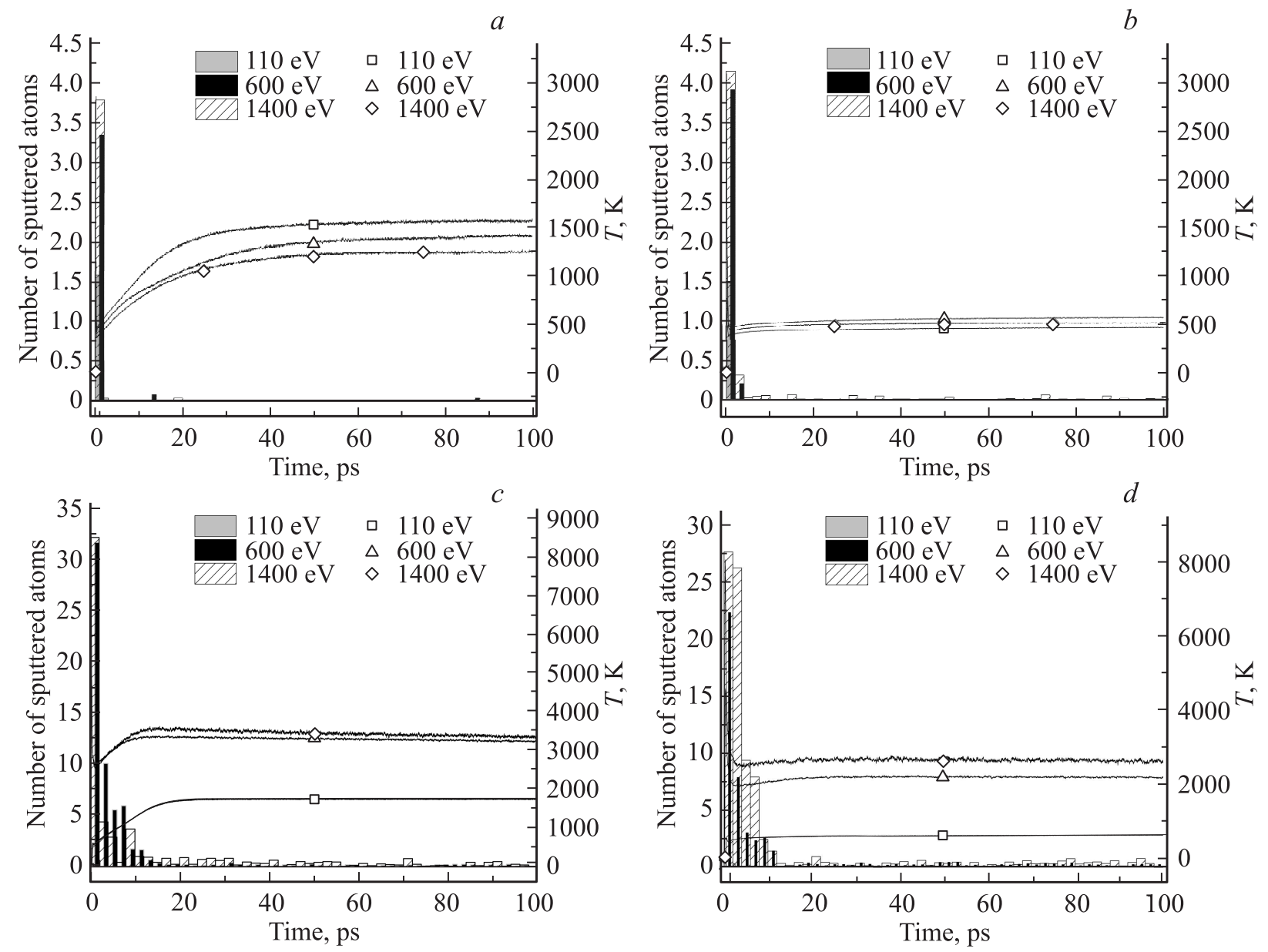

Рис. 2. Зависимость температуры и выхода распыления двудольных кластеров от времени для различных начальных энергий и типов бомбардирующей частицы: $\left.a) \mathrm{Ar}, \mathrm{Ni}-\mathrm{Al} ; b) \mathrm{Ar}, \mathrm{Cu}-\mathrm{Au} ; c) \mathrm{Ar}_{13}, \mathrm{Ni}-\mathrm{Al} ; d\right) \mathrm{Ar}_{13}, \mathrm{Cu}-\mathrm{Au}$.

распылением некоторых из них. В случае кластера $\mathrm{Cu}-\mathrm{Au}$ температура стабилизируется за первые 5-10 ps существенно ниже точек плавления макроскопических образцов $\mathrm{Cu}$ и $\mathrm{Au}$, тогда как для кластера $\mathrm{Ni}-\mathrm{Al}$ стабилизация наступает после $40-50$ ps и температура устанавливается незначительно ниже точки плавления $\mathrm{Ni}$, но существенно выше точки плавления Al. При этом, процесс массопереноса на границе раздела компонентов, а также в глубине долей кластера на более поздних стадиях, продолжается в течение всего времени моделирования, хотя его интенсивность существенно ниже, чем в случае бомбардировки кластерами $\mathrm{Ar}_{13}$.

При бомбардировке кластерами $\mathrm{Ar}_{13}$ с энергиями выше $100 \mathrm{eV}$ тоже наблюдается резкий пик температуры в течение первой пикосекунды. Далее в течение последующих 1-3 ps в окрестностях минимума температуры происходит развитие столкновительного каскада в ограниченном объеме кластера за счет привнесенной энергии бомбардирующей частицы. Этот этап характеризуется конкурирующими процессами, регулирующими температуру кластера, а именно распылением низкоэнергетических атомов, с одной стороны, и экзотермическим перемешиванием атомов компонентов с одновременной потерей двудольной формы кластера, приводящей к сокращению свободной поверхности [48] - с другой. По мере рассеяния привнесенной кластером $\mathrm{Ar}_{13}$ энергии в ограниченном объеме бомбардируемого кластера и связанного с ним затухания каскадно-рекойлового столкновительного процесса, а также выделения энергии в результате перемешивания и потери двудольной формы, температура кластера повышается и в окрестностях последующих 1-2 ps на кривой температуры наблюдается перегиб, после которого доминирующим становится процесс перемешивания компонентов за счет выросшей к данному моменту времени температуры кластера. Попутно, кластер теряет кристаллическую ГЦК-структуру долей, что особенно сильно проявляется в кластере $\mathrm{Ni}-\mathrm{Al}$ уже в интервале 5-10 ps с начала взаимодействия при начальной энергии бомбардировки выше $100 \mathrm{eV}$. При больших энергиях бомбардировки это происходит еще быстрее. Подобная конкуренция процессов, регулирующих температуру, происходит и при бомбардировке единичными атомами Ar, однако в пределах более короткого временно́го интервала, определяемого спецификой возникающего каскадно-рекойлового распыления. 
Повышение температуры кластера за счет экзотермического перемешивания в случае бомбардировки кластерами $\mathrm{Ar}_{13}$ продолжается до 20-25 ps с начала столкновительного взаимодействия. Следует отметить, что увеличение температуры кластера за счет отрицательной теплоты перемешивания в случае кластера $\mathrm{Cu}-\mathrm{Au}$ сравнимо с вкладом в изменение температуры кластера уменьшения свободной поверхности при слиянии двудольного кластера в единый кластер. В случае кластера $\mathrm{Ni}-\mathrm{Al}$ роль уменьшения свободной поверхности оказывается незначительной по сравнению с эффектом перемешивания компонентов. После 25 рs эволюции кластера наступает период стабилизации температуры, при которой в случае кластера $\mathrm{Ni}-\mathrm{Al}$, температура стабилизируется выше точки кипения объемных образцов никеля и алюминия при энергиях бомбардировки больше $300 \mathrm{eV}$, а в случае кластера $\mathrm{Cu}-\mathrm{Au}$ температура на участке стабилизации оказывается существенно выше точек плавления меди и золота. Для обоих кластеров в данных условиях моделирования наблюдается испарение атомов с поверхности и уменьшение их температуры, причем для кластера $\mathrm{Ni}-\mathrm{Al}$ испарение протекает интенсивнее.

Таким образом, временной интервал до 5 ps c начала столкновительного взаимодействия характеризуется изменяющейся во времени активностью механизмов массопереноса различной природы и структурных изменений вблизи границы раздела долей кластера, управляемость которыми во многом определяется типом (единичный атом/атомный кластер) и энергией бомбардирующих частиц. Дальнейшая реальная эволюция бомбардируемых кластеров не ограничивается выбранными временны́ми рамками моделирования до 100 ps и в основном определяется выделяющейся при перемешивании компонентов и сокращении свободной поверхности энергией, а также испарением атомов. Это в значительной степени маскирует исходную роль выбора условий бомбардировки и добавляет актуальности молекулярнодинамическим исследованиям начального этапа эволюции двудольных кластеров. Согласно вышеуказанному, начальный этап определяется как первые 5 ps, и все последующие модельные исследования относятся к этому моменту времени.

На рис. 3 показаны относительные потенциальные энергии бинарных кластеров, которые устанавливаются по истечении 5 ps с момента начала его взаимодействия с налетающим кластером $\mathrm{Ar}_{n}$ в зависимости от исходной энергии последнего. Потенциальные энергии нормированы на модуль начальной потенциальной энергии металлического кластера. Видно, что размер налетающего кластера значительно влияет на потенциальную энергию мишени, что объясняется увеличением средних расстояний между атомами в бомбардируемом кластере с ростом его температуры (см. ниже рис. 4), а также сопутствующими эффектами нарушения порядка и распыления. Данные о бомбардировке димерами $\mathrm{Ar}_{2}$ не приведены на рис. 3, поскольку они показывают только незначительно бо́льшую потенциальную энергию двудольного кластера по сравнению со случаем единичных

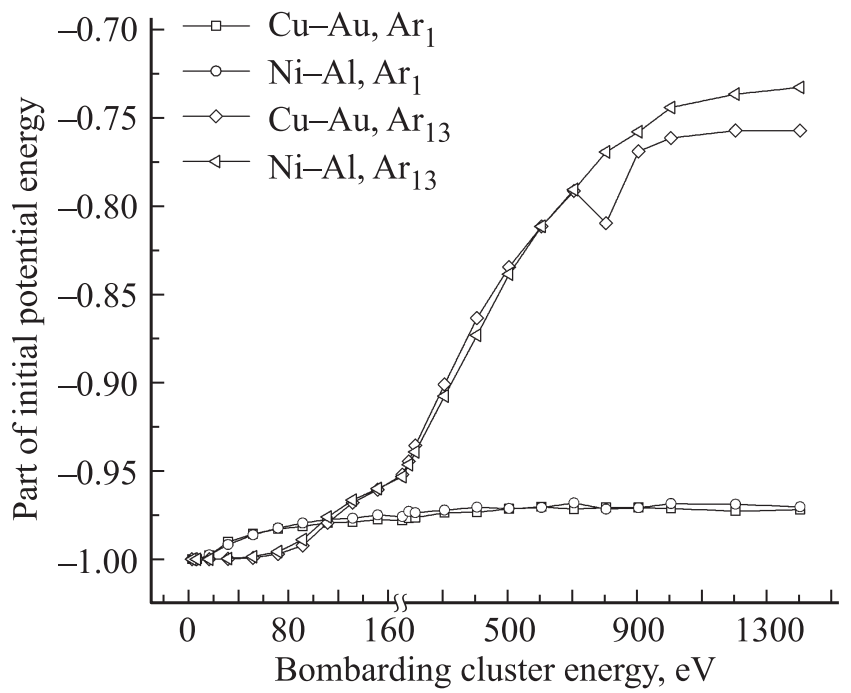

Рис. 3. Зависимость потенциальной энергии мишени от энергии и типа бомбардирующего кластера ${ }^{1}$.

атомов $\mathrm{Ar}$ и соответственно существенно меньший рост потенциальной энергии на фоне результатов для кластеров $\mathrm{Ar}_{13}$ при энергиях бомбардировки более $200 \mathrm{eV}$.

При энергиях бомбардировки до $120 \mathrm{eV}$, потенциальная энергия мишени в случае налетающих частиц $\mathrm{Ar}_{13}$ ниже, чем при бомбардировке единичными атомами $\mathrm{Ar}$ и димерами $\mathrm{Ar}_{2}$. Для большего кластера аргона энергия в расчете на один атом меньше, чем при бомбардировке мономерами и димерами, что уменьшает глубину проникновения атомов кластера аргона в мишень, и при низкой общей энергии первичные столкновительные процессы инициируются преимущественно в ее приповерхностных слоях. Тем самым меньшему числу преимущественно поверхностных атомов мишени энергия передается непосредственно от атомов налетающего кластера, и ее потенциальная энергия изменяется незначительно в результате малых деформаций атомной структуры. При этом большая доля энергии (по сравнению со случаями более высоких энергий бомбардировки) передается двудольному кластеру как цельному телу, заставляя его центр масс совершать поступательное движение.

На рис. 4 представлен график зависимости температуры биметаллического кластера от энергии бомбардирующих частиц после 5 ps моделирования. Видно, что изменения температуры и, как показано на предыдущем рисунке, потенциальной энергии демонстрируют качественно одинаковый характер. Для всех бомбардирующих кластеров аргона температура кластера $\mathrm{Ni}-\mathrm{Al}$ больше температуры кластера $\mathrm{Cu}-\mathrm{Au}$, как уже было показано в [34] для случая налетающих мономеров аргона. Большей максимальной температуре для кластера $\mathrm{Ni}-\mathrm{Al}$ способствует бо́льшая эффективность передачи

\footnotetext{
1 Здесь и далее разрыв на оси абсцисс означает точку изменения масштаба.
} 


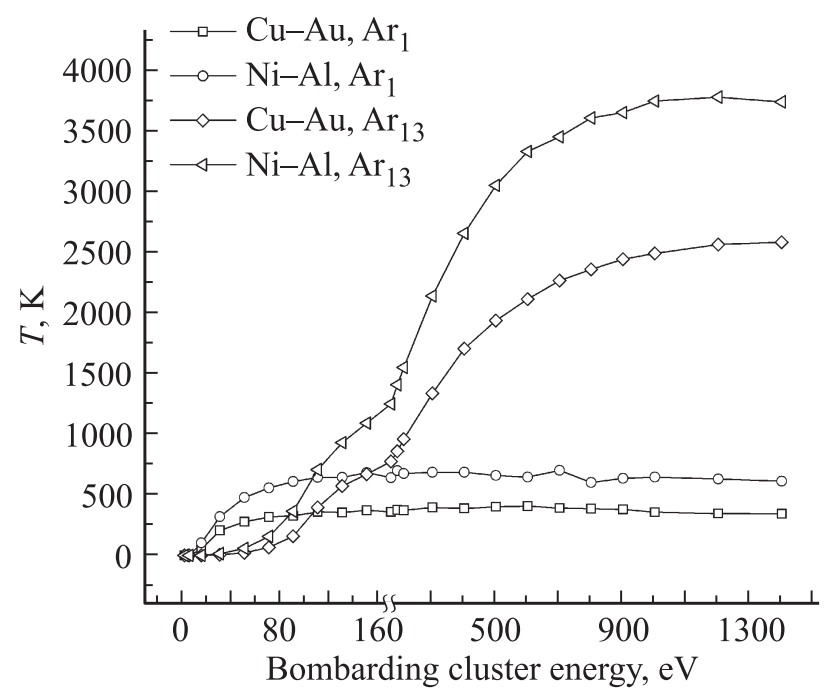

Рис. 4. Зависимость температуры бинарного кластера от энергии и размера бомбардирующего кластера.

энергии при первичных столкновениях его атомов обоих типов с атомами аргона, чем для взаимодействий $\mathrm{Au}-\mathrm{Ar}$ в кластере $\mathrm{Cu}-\mathrm{Au}$. Кроме того, с таким результатом моделирования согласуется и меньшая теплота перемешивания для $\mathrm{Ni}-\mathrm{Al}$, о чем уже упоминалось выше. Были получены следующие максимальные температуры нагрева бинарных кластеров к концу 5 ps: 406, 637, $2562 \mathrm{~K}$ для $\mathrm{Cu}-\mathrm{Au}$ и $700,958,3754 \mathrm{~K}$ для $\mathrm{Ni}-\mathrm{Al}$ при бомбардировке кластерами $\mathrm{Ar}_{1}, \mathrm{Ar}_{2}, \mathrm{Ar}_{13}$ соответственно. Очевидно, что для большего бомбардирующего кластера при равных и не слишком низких начальных энергиях, о поведении кластера-мишени при которых также указывалось выше, определяющим фактором являются бо́льшие времена взаимодействия атомов аргона с мишенью и соответственно возможности передачи энергии отдельным атомам мишени тоже больше, чем в случае бомбардирующего димера или мономера аргона.

Зависимости выходов распыления от энергии налетающих кластеров $\operatorname{Ar}_{n}$ за первые 5 ps эволюции двудольных кластеров представлены на рис. 5. Исходя из данных, представленных на рис. 2, к концу пятой пикосекунды заканчивается определяемая работой каскадно-рекойлового столкновительного механизма стадия интенсивного распыления, что наиболее четко проявляется при бомбардировке единичными атомами Ar, и постепенно активизируется работа термического механизма, который в случае достаточных начальных энергий бомбардировки проявляет себя в течение всего времени моделирования. Как видно из рис. 5, для случаев бомбардировки ионами $\mathrm{Ar}$ и $\mathrm{Ar}_{13}$, а также следует из отсутствующих на рисунке данных для $\mathrm{Ar}_{2}$, выходы распыления возрастают с увеличением размера бомбардирующего кластера при высоких энергиях. Это качественно согласуется с синергетическим эффектом увели- чения выхода распыления из поверхностных кластеров меди при бомбардировке одноименными атомными димерами по сравнению с удвоенным выходом распыления, инициированным единичными одноименными атомами с половинными начальными энергиями [49]. При низких энергиях бомбардировки меньшие выходы распыления для больших бомбардирующих кластеров объясняются малыми энергиями в расчете на один бомбардирующий атом, которые не обеспечивают возможностей для разворота импульсов с передачей необходимых энергий атомам бомбардируемого кластера или инициирования в нем сквозных атомных цепочек замещения.

На рис. 5, $a$ выход распыления атомов $\mathrm{Cu}$ превосходит выход распыления атомов Аu во всех моделируемых случаях, что качественно согласуется с общепризнан-
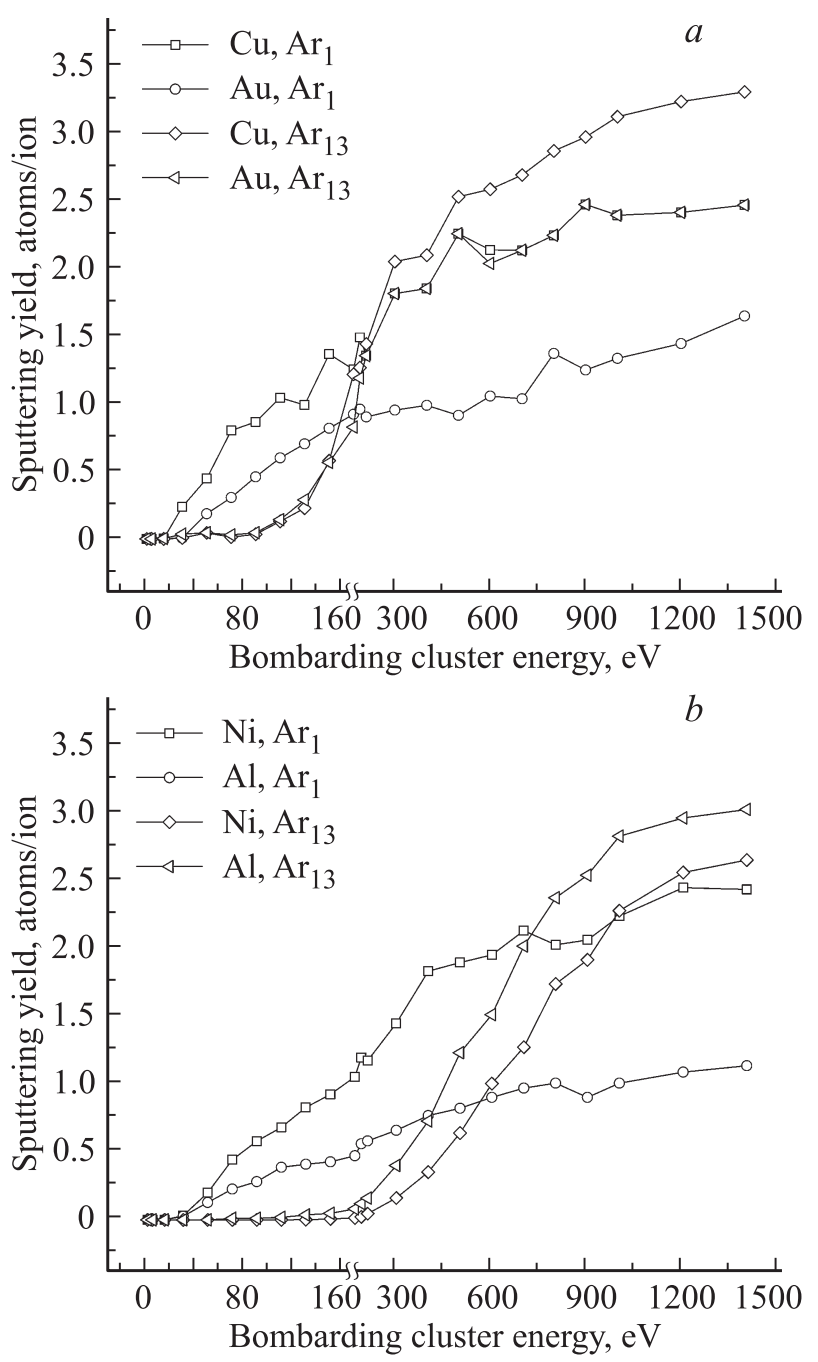

Рис. 5. Выходы распыления монокомпонентных частей кластера $\mathrm{Cu}-\mathrm{Au}(a)$ и $\mathrm{Ni}-\mathrm{Al}(b)$ для различных энергий и размеров бомбардирующих кластеров аргона. Выходы распыления представлены в расчете на один атом Ar, т.е. полный выход распыления для случая $\operatorname{Ar}_{13}$ вычисляется путем умножения значения выхода распыления на графике на 13. По оси абсцисс отложены начальные энергии бомбардирующих частиц $\mathrm{Ar}$ и $\operatorname{Ar}_{13}$. 

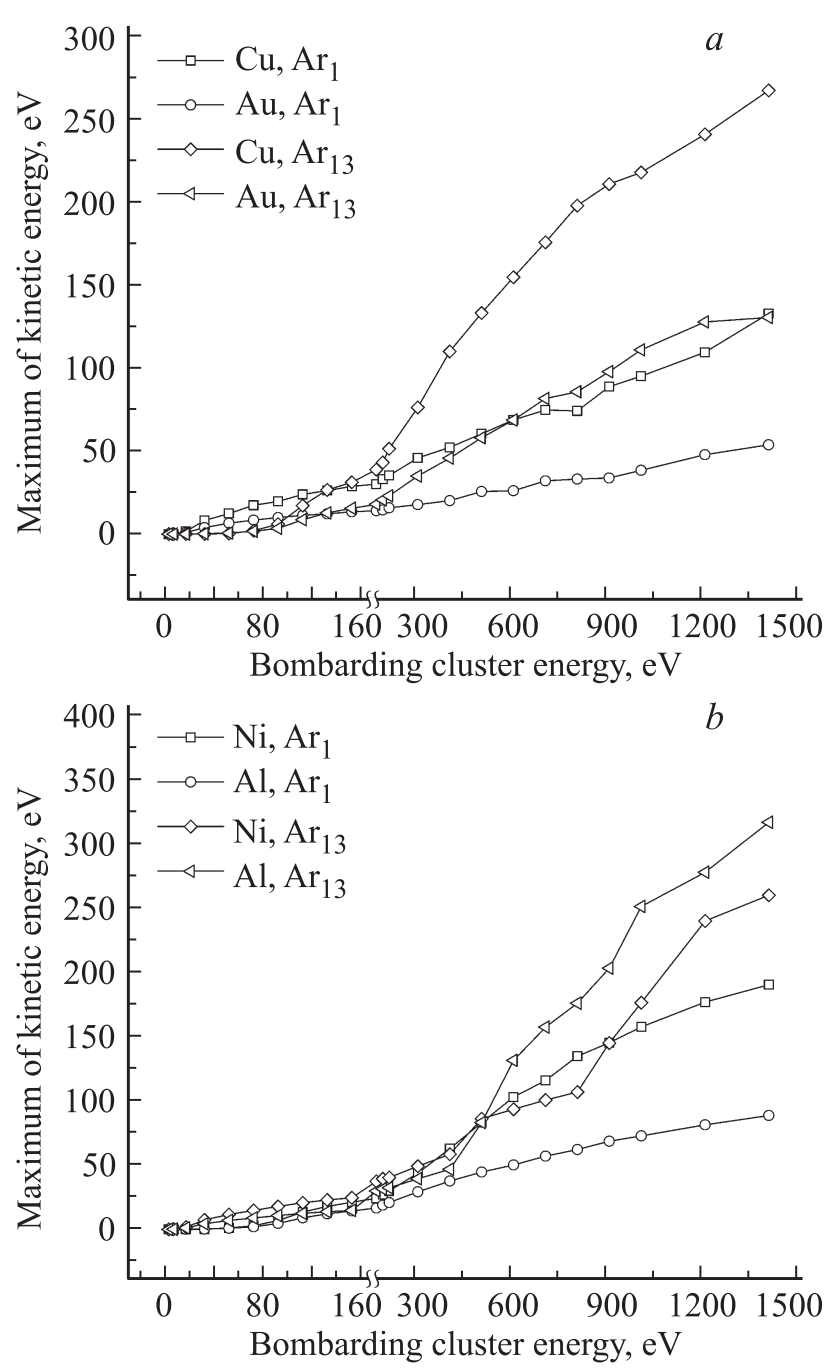

Рис. 6. Максимальные кинетические энергии монокомпонентных частей кластера $\mathrm{Cu}-\mathrm{Au}(a)$ и $\mathrm{Ni}-\mathrm{Al}(b)$ для различных энергий и размеров бомбардирующих кластеров аргона.

ными теоретическими представлениями и экспериментальными результатами для макроскопических образцов соответствующих элементов [50,51] и определяется в первую очередь различием эффективностей передачи энергии атомам $\mathrm{Cu}$ и $\mathrm{Au}$ налетающими атомами $\mathrm{Ar}$. Это в свою очередь согласуется с данными по максимальным кинетическим энергиям однокомпонентных долей бинарных кластеров, приведенным для случая $\mathrm{Cu}-\mathrm{Au}$ на рис. 6,a. Для кластера $\mathrm{Ni}-\mathrm{Al}$ при бомбардировке одноатомными частицами Ar большее количество распыленных частиц приходится на никель [52], тогда как при бомбардировке многоатомными кластерами аргона доминирует распыление алюминия (рис. $5, b$ ). В отличие от кластера $\mathrm{Cu}-\mathrm{Au}$, атомы $\mathrm{Ar}$ по отношению к атомам $\mathrm{Al}$ и $\mathrm{Ni}$ имеют практически одинаковые эффективности передачи энергии. В данном случае следует обратиться к объяснению, предложенному в [52], а именно, что одноатомные бомбардирующие частицы проникают с меньшими потерями энергии сквозь более прозрачную атомную структуру (см. раздел 2) алюминиевой части и интенсивнее теряют энергию в никелевой части кластера [53]. Многоатомные кластеры аргона не обладают достаточной энергией в расчете на один атом $\mathrm{Ar}$ и пространственной локализацией самих атомов $\mathrm{Ar}$ для проникновения сквозь структуру алюминиевой части кластера и активации распылительных процессов в никелевой части. В итоге взаимодействие кластера аргона с алюминиевой долей бинарного кластера носит коллективный характер, приводит к рассеиванию входящей энергии в этой доле, разрушению ее атомной структуры и росту выхода распыления алюминия. Это подтверждается значениями максимальной кинетической энергии для каждой доли кластера, которую можно рассматривать, как оценку энергии, передаваемой бомбардирующими частицами соответствующей части мишени (см. рис. 6, $b$ ), а также согласуется с тем, что поверхностная энергия связи алюминия существенно меньше, чем никеля. Следует также отметить, что полученные путем моделирования выходы распыления для кластеров в случае одноатомных бомбардирующих частиц традиционно больше приведенных в [50] экспериментальных выходов распыления для плоских макроскопических образцов соответствующих элементов, чему неоднократно давались объяснения в других работах [21-27,54,55].

C ростом энергии бомбардировки кластера $\mathrm{Ar}_{13}$ выше $300 \mathrm{eV}$ к столкновительным механизмам распыления благодаря очень высоким температурам в металлическом кластере, как видно из рис. 2 и 4, добавляется испарение атомов с его поверхности. Очевидно, этот механизм вносит существенный вклад в изменение преимущественно распыляемого элемента кластера $\mathrm{Al}-\mathrm{Ni}$ с ростом размера бомбардирующего кластера, а именно в распыление алюминиевой доли. Этому способствует низкая по сравнению с другими моделируемыми компонентами температура плавления алюминия, которая для кластера небольшого размера может быть еще ниже. Как и для одноатомных частиц, при бомбардировке кластерами удаление атомов происходит преимущественно из приповерхностной области металлического кластера.

На рис. 7 показано усредненное по числу испытаний количество распыленных мономеров, димеров и тримеров в случае бинарного кластера $\mathrm{Cu}-\mathrm{Au}$. Для случаев бомбардировки частицами $\mathrm{Ar}_{1}$ и $\mathrm{Ar}_{2}$ в распыленном материале преобладают единичные атомы, хотя и вклады димеров $\mathrm{Cu}_{2}$ не являются пренебрежимо малыми, тогда как относительные вклады димеров $\mathrm{Au}_{2}$ существенно меньше (рис. 7, $a, b$ ). В случае $\mathrm{Ar}_{13}$ при энергиях больше $200 \mathrm{eV}$ заметно преобладают атомы, распыленные в составе димеров $\mathrm{Cu}_{2}$, а выходы распыления в виде мономеров $\mathrm{Au}$ и димеров $\mathrm{Au}_{2}$ сравнимы между собой для различных энергий бомбардировки. Это, очевидно, также является следствием высоких температур металлического кластера (см. рис. 4 и 7,c), поскольку при возникновении высокой температуры снижается энергетический порог выхода распыления. Также повышается 

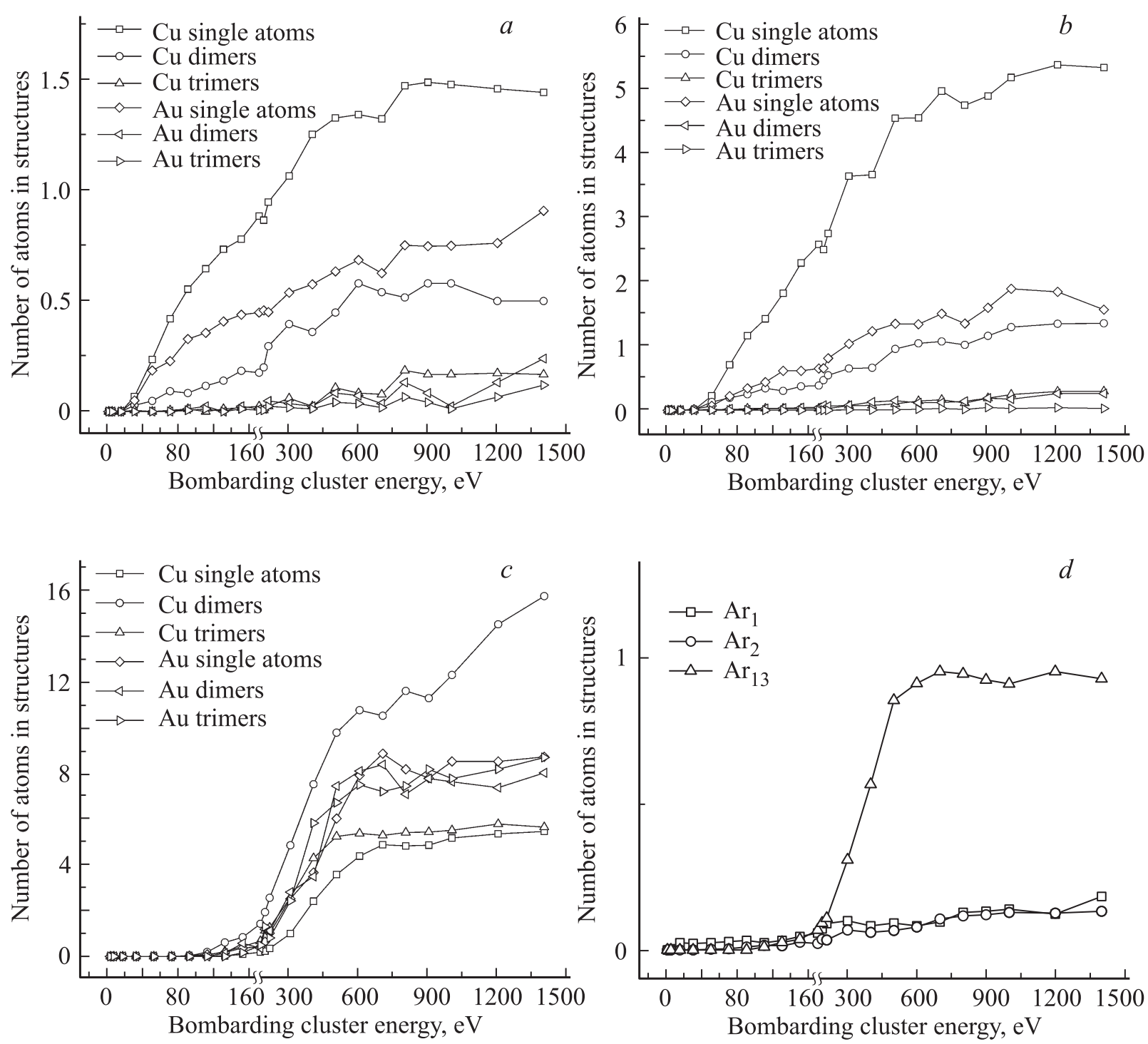

Рис. 7. Число атомов в многоатомных фрагментах распыленного материала для кластера $\mathrm{Cu}-\mathrm{Au}$ при различных энергиях бомбардировки. $a-\mathrm{Ar}_{1}, b-\mathrm{Ar}_{2}, c-\mathrm{Ar}_{13}, d-$ димеры $\mathrm{Cu}-\mathrm{Au}$.

вероятность одновременного получения достаточных и соответствующих друг другу по величине нормальных к поверхности кластера компонентов импульсов соседними поверхностными атомами мишени (подобный механизм распыления димера, но без учета роли температуры описан в [56]), и их дальнейшего распыления в виде димеров. При бомбардировке кластерами $\mathrm{Ar}_{13}$ с энергией больше $200 \mathrm{eV}$ также увеличивается количество более сложных распыленных структур, в частности тримеров, а также отсутствующих на рисунке, но идентифицированных при моделировании, тетрамеров. Увеличивается также количество распыленных биметаллических структур, как показано на рис. 7, $d$ для случая димеров $\mathrm{Cu}-\mathrm{Au}$. Аналогично в случае кластера $\mathrm{Ni}-\mathrm{Al}$ значительно увеличивается выход атомов в виде димеров. При этом преобладают димеры алюминия. Следует отметить также проявление порогового харак- тера выхода распыления на рис. 5 и 7, $a-c$ в виде близких к нулю участков выходов распыления, оценочно соответствующих энергии до $20 \mathrm{eV}$ в расчете на один бомбардирующий атом.

Также отслеживалось количество перемещений поверхностных и внутренних атомов из одной доли кластера в другую за время моделирования столкновительного процесса 5 ps (рис. 8). Большее количество перескоков атомов из поверхностного слоя для $\mathrm{Al}$ и в меньшей степени для Аu объясняется большими геометрическими размерами соответствующих долей исходных кластеров в плоскости соприкосновения монометаллических частей, что способствует перемещению атомов $\mathrm{Al}$ на поверхность $\mathrm{Ni}$, а атомов $\mathrm{Au}$ на поверхность $\mathrm{Cu}$. Следует отметить высокое суммарное количество перескоков для кластера $\mathrm{Ni}-\mathrm{Al}$ по сравнению с кластером $\mathrm{Cu}-\mathrm{Au}$. Это обстоятельство связано с тем, что теплота перемешива- 

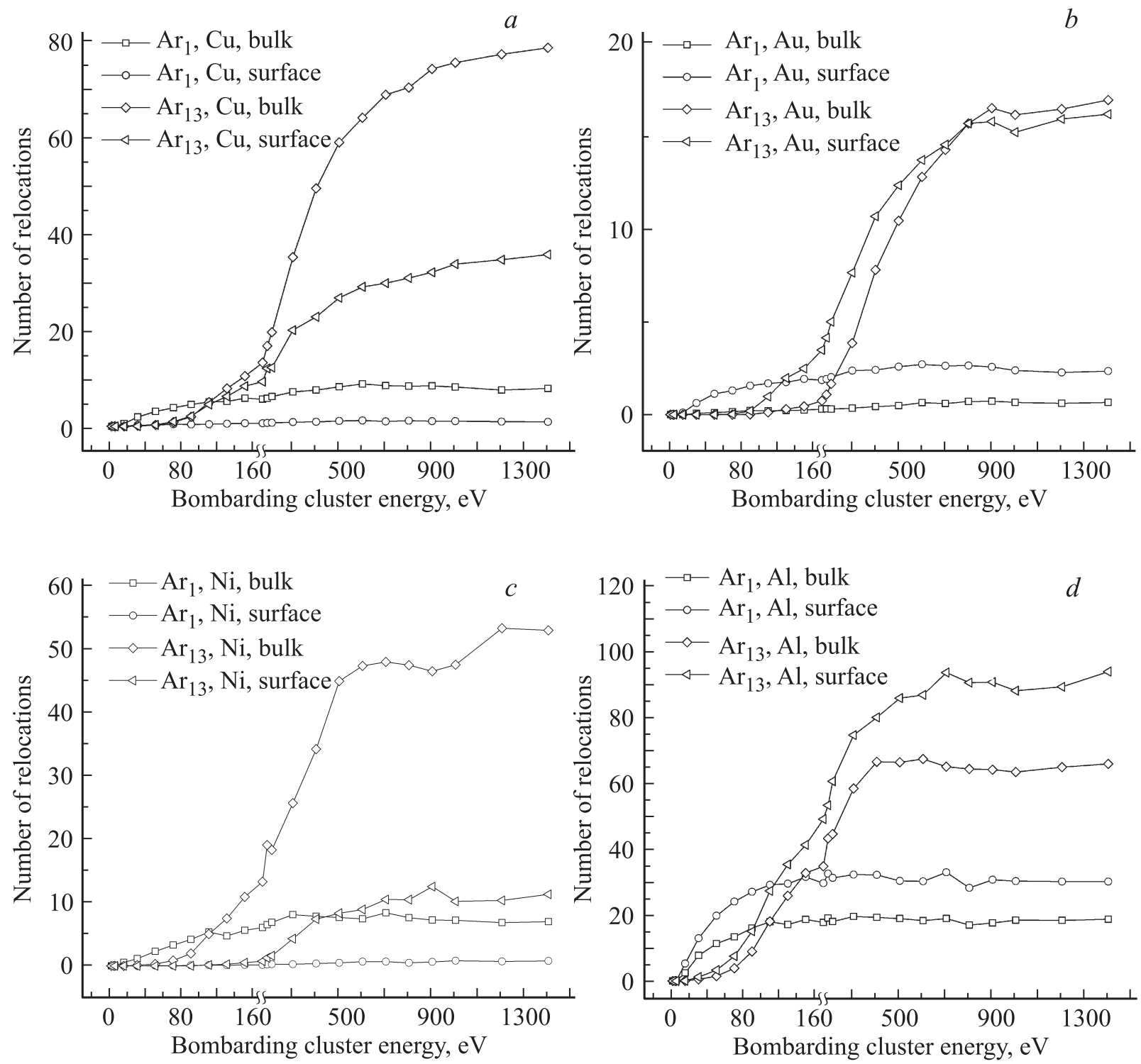

Рис. 8. Зависимость количества перескоков атомов $\mathrm{Cu}(a), \mathrm{Au}(b), \mathrm{Ni}(c), \mathrm{Al}(d)$ через границу раздела монокомпонентных долей в металлическом кластере от энергии и размера бомбардирующего кластера аргона.

ния пары $\mathrm{Ni}-\mathrm{Al}$ ниже, чем $\mathrm{Cu}-\mathrm{Au}$. Кроме того, тяжелые атомы $\mathrm{Au}$ имеют значительно меньшую подвижность по сравнению с легкими атомами $\mathrm{Al}$, которые вносят доминирующий вклад в общее количество перемещений через границу раздела компонентов соответствующего бинарного кластера. Также наблюдается резкий рост количества перемещений через границу раздела в обоих кластерах при энергиях кластеров $\mathrm{Ar}_{13}$ больше $200 \mathrm{eV}$, что очевидно связано с увеличением температуры по причинам, описанным выше. Для атомов элементов с меньшей постоянной решетки $(\mathrm{Cu}, \mathrm{Ni})$ для каждого типа бомбардирующего кластера преобладает движение внутренних атомов в составе бинарных двудольных кластеров. В целом же видно, что подвижность атомов повышается с увеличением размера бомбардирующего кластера.

\section{4. Заключение}

Выполнено моделирование эволюции в течение 5 и 100 ps биметаллических двудольных кластеров $\mathrm{Cu}-\mathrm{Au}$ и $\mathrm{Ni}-\mathrm{Al}$ при бомбардировке частицами $\mathrm{Ar}_{1}, \mathrm{Ar}_{2}, \mathrm{Ar}_{13}$ c различными начальными энергиями. Моделирование в течение $100 \mathrm{ps}$ позволило получить эволюцию температуры кластеров, установить значительность вклада термического распыления атомов в общем выходе распыления и постепенном охлаждении кластеров. Изучение состояния кластеров к моменту времени $5 \mathrm{ps}$ дало возможность оценить роль каскадно-рекойловых столкновительных механизмов в их эволюции, а также рассмотреть начальный этап развития термической стадии массопереноса. Получена высокая энергоотдача при низкоэнергетической бомбардировке кластерами 
аргона двудольных кластеров, позволяющая активировать в них интенсивные процессы перемешивания и распыления.

Показано, что изменения потенциальной энергии и температуры металлического кластера за 5 ps эволюции имеют качественно иную форму при бомбардировке кластерами $\mathrm{Ar}_{13}$ по сравнению с $\mathrm{Ar}_{1}$ и $\mathrm{Ar}_{2}$, что связано с низкими энергиями в расчете на один бомбардирующий атом. Температура кластера $\mathrm{Ni}-\mathrm{Al}$ выше температуры кластера $\mathrm{Cu}-\mathrm{Au}$ при всех условиях бомбардировки, что объясняется особенностями передачи энергии от атомов Ar атомам различных элементов металлических кластеров и меньшей теплотой перемешивания $\mathrm{Ni}-\mathrm{Al}$ по сравнению с $\mathrm{Cu}-\mathrm{Au}$.

Расчет выходов распыления за 5 рs эволюции двудольных кластеров показывает рост количества распыленных единичных атомов, а также многоатомных фрагментов включая бикомпонентные, с увеличением размера бомбардирующего кластера, чему способствует критическое увеличение при этом температуры двудольных кластеров. Имеет место преимущественное распыление атомов $\mathrm{Ni}$ при бомбардировке кластера $\mathrm{Ni}-\mathrm{Al}$ одноатомными частицами Ar, тогда как в случае бомбардирующих частиц $\mathrm{Ar}_{2}$ и $\mathrm{Ar}_{13}$ происходит преимущественное распыление атомов Al. В случае же кластера $\mathrm{Cu}-\mathrm{Au}$ наблюдается ожидаемое преимущественное распыление атомов $\mathrm{Cu}$ по сравнению с $\mathrm{Au}$ при всех условиях моделирования. В целом происходит больше перемещений атомов в кластере $\mathrm{Ni}-\mathrm{Al}$ при всех модельных условиях бомбардировки.

Полученные результаты работы могут быть использованы при разработке процессов магнетронного напыления тонкопленочных наноструктурированных слоев $\mathrm{Ni}-\mathrm{Al}$ или $\mathrm{Cu}-\mathrm{Au}$ на элементы полупроводниковых структур для обеспечения их эффективного сращивания при невысоких температурах с обеспечением высоких теплопроводящих и прочностных характеристик. В частности, изготовление таких активных слоев необходимо для реализации вертикальной геометрии сборки полупроводниковых преобразователей бета-излучения в электрическую энергию на основе диодных структур из синтетических алмазов.

Численные расчеты выполнялись на высокопроизводительном вычислительном кластере ЦКП ФГБНУ ТИСНУМ (Троицк, Москва).

\section{Список литературы}

[1] M. Fárník. Molecular Dynamics in Free Clusters and Nanoparticles. Institute Chem. Technology, Prague (2011). 98 p.

[2] T. Rapps, R. Ahlrichs, E. Waldt, M.M. Kappes, D. Schooss. Angew. Chemie-Int. Ed. 52, 6102 (2013).

[3] M. Huttula, M.H. Mikkelä, M. Tchaplyguine, O. Björneholm. J. Electron Spectr. Rel. Phenomena 181, 145 (2010).

[4] X. Wu, W. Cai, X. Shao. J. Comp. Chem. 30, 1992 (2009).

[5] F. Yin, Z.W. Wang, R.E. Palmer. J. Exp. Nanosci. 7, 703 (2012).
[6] S. Darby, T.V. Mortimer-Jones, R.L. Johnston, C. Roberts. J. Chem. Phys. 116, 1536 (2002).

[7] M. Calleja, C. Rey, M. Alemany, L. Gallego, P. Ordejón, D. Sánchez-Portal, E. Artacho, J. Soler. Phys. Rev. B 60, 2020 (1999).

[8] B.-X. Li, X. Ren, X. Zhang, Z. Ma, J. Gu, G. Li. World J. Condens. Matter Phys. 02, 267 (2012).

[9] E.C. Tyo, S. Vajda. Nature Nanotechnol. 10, 577 (2015).

[10] Z.A. Qiao, P. Zhang, S.H. Chai, M. Chi, G.M. Veith, N.C. Gallego, M. Kidder, S. Dai. J. Am. Chem. Soc. 136, 11260 (2014).

[11] R. Ferrando, J. Jellinek, R.L. Johnston. Chem. Rev. 108, 845 (2008).

[12] A.J. Gavens, D. Van Heerden, A.B. Mann, M.E. Reiss, T.P. Weihs. J. Appl. Phys. 87, 1255 (2000).

[13] M.D. Wiemer, J. Braeuer, D. Wünsch, T. Gessner. ECS Trans. 33, 307 (2010).

[14] J. Braeuer, J. Besser, M. Wiemer, T. Gessner. 16th Int. Solid-State Sensors, Actuators Microsystems Conf., Beijing, China IEEE (2011). P. 1332.

[15] F. Weigend, R. Ahlrichs. Philos. Trans. A. Math. Phys. Eng. Sci. 368,1245 (2010).

[16] T. Mazza, M. Devetta, P. Milani, G. Bongiorno, M. Coreno, P. Piseri. New J. Phys. 13, 023009 (2011).

[17] Y. Lu, W. Chen. Chem. Soc. Rev. 41, 3594 (2012).

[18] C. Gang, Z. Peng, L. Hongwei. Adv. Mater. Sci. Eng. 2014, 1 (2014).

[19] O.A. Yermolenko, G.V. Kornich. J. Surf. Investig. X-Ray, Synchrotron Neutron Tech. 6, 222 (2012).

[20] I. Hamid, M. Fang, H. Duan. AIP Adv. 5, 047129 (2015).

[21] R. Kissel, H.M. Urbassek. Nucl. Instr. Meth. B 180, 293 (2001).

[22] T.T. Järvi, J.A. Pakarinen, A. Kuronen, K. Nordlund. Eur. Lett. 82, 2 (2008).

[23] S. Zimmermann, H.M. Urbassek. Int. J. Mass Spectr. 272, 1, 91 (2008).

[24] T.T. Järvi, A. Kuronen, K. Nordlund. Phys. Rev. B 80, 132101 (2009).

[25] T.T. Järvi, K. Nordlund. Nucl. Instr. Meth. B 272, 1, 66 (2012).

[26] M.L.Nietiadi, L. Sandoval, H.M. Urbassek, W. Möller. Phys. Rev. B 90, 045417 (2014).

[27] L. Sandoval, H.M. Urbassek. Nanoscale Res. Lett. 10, 314 (2015).

[28] I. Yamada, J. Matsio, N. Toyoda. Nucl. Instr. Meth. Phys. Res. B 206, 820 (2003).

[29] I. Yamada. Radiat. Eff. Defects Solids 124, 69 (1992).

[30] N. Toyoda, I. Yamada. Phys. Procedia 66, 556 ( 2015).

[31] A. Delcorte, E. Pospisilova, C. Poleunis, V. Cristaudo. 22th Int. Ion-Surface Interactions Conf. Moscow, ISI (2015). V. 1. P. 22.

[32] A.Y. Galashev, O.R. Rakhmanova. Chin. Phys. B 24, 2, 020701 (2015).

[33] V.S. Chernysh, A.E. Ieshkin, Yu.A. Ermakov. Appl. Surf. Sci. 326, 285 (2015).

[34] Д.В. Широкорад, Г.В. Корнич, С.Г. Буга. ФТТ 58, 377 (2016).

[35] A. Takeuchi, A. Inoue. Mater. Trans. 46, 2817 (2005).

[36] S. Hendy, S.A. Brown, M. Hyslop. Phys. Rev. B 68, 241403(R) (2003).

[37] Д.В. Широкорад, Г.В. Корнич. ФТТ 56, 2475 (2014).

[38] J. Gibson, A. Goland, M. Milgram, G. Vineyard. Phys. Rev. 120, 1229 (1960). 
[39] M.W. Finnis, J.E. Sinclair. Phil. Mag. A 50, 45 (1984).

[40] V. Vitek, G.J. Ackland, J. Cserti. MRS Proc. 186, 237 (1990).

[41] T. Uchida, H. Matsuoka, H. Matsui, M. Doyama. Structural Fluctuations in Gold Microclusters. In: Computer Aided Innovation of New Materials / Eds M. Doyama, T. Suzuki, J. Kihara, R. Yamamoto. Elsevier B.V, 371 (1991).

[42] E.E. Zhurkin, M. Hou. J. Phys.: Condens. Matter 12, 6735 (2000).

[43] E.E. Zhurkin, G. Hautier, M. Hou. Phys. Rev. B 73, 094108 (2006).

[44] J.F. Ziegler, M.D. Ziegler, J.P. Biersack. Nucl. Instr. Methods Phys. Res. B 268, 1818 (2010).

[45] R.A. Aziz, M.J. Slaman. Zeitschrift Für Phys. D 25, 343 (1993).

[46] R.A. Aziz, H.H. Chen. J. Chem. Phys. 67, 5719 (1977).

[47] W. Eckstein. Computer Simulation of Ion-Solid Interactions. Springer-Verlag Berlin-Heidelberg (2011). 296 p.

[48] J. Antunez-Garcia, S. Mejia-Rosales, E. Perez-Tijerina, J.M. Montejano-Carrizales, M. Jose-Yacaman. Materials 4, 368 (2011).

[49] G.V. Kornich, G. Betz, V.G. Kornich, V.I. Shulga, O.A. Yermolenko. Nucl. Instruments Methods Phys. Res. B 269, 1600 (2011).

[50] Sputtering by Particle Bombardment / Eds R. Behrisch, W. Eckstein. Springer Berlin-Heidelberg, Berlin, Heidelberg (2007).

[51] Sputtering by Particle Bombardment II / Ed. R. Behrisch. Springer-Verlag Berlin-Heidelberg (1983).

[52] G.V. Kornich, G. Betz. Nucl. Instruments Methods Phys. Res. B 143, 455 (1998).

[53] G.V. Kornich, G. Betz, A.I. Bazhin. Nucl. Instruments Methods Phys. Res. B 153, 383 (1999).

[54] G.V. Kornich, G. Betz, V. Zaporojtchenko, A.I. Bazhin, F. Faupel. Nucl. Instruments Methods Phys. Res. B 227, 261 (2005).

[55] G.V. Kornich, G. Betz, V. Zaporojtchenko, K.V. Pugina. Surf. Sci. 601, 209 (2007).

[56 ] G. Betz, K. Wien. Int. J. Mass Spectrom. Ion Process. 140, 1 (1994). 